\title{
Contactless Manipulation of Microparts by ac Electric Fields
}

\author{
Felix M. Moesner ${ }^{1,2)}$ and Toshiro Higuchi ${ }^{2,3)}$ \\ 1) Swiss Federal Institute of Technology Zurich (ETHZ) • Institute of Robotics • 8092 Zurich, Switzerland \\ Tel: +411632 3582•Fax: +411632 1078•Email: moesner@ifr.mavt.ethz.ch \\ 2) Kanagawa Academy of Science and Technology • Higuchi 'Ultimate Mechatronics' Project \\ KSP Bldg., East 405, 3-2-1 Sakado, Takatsu-ku, Kawasaki 213, Japan • Fax: +81 448192095 \\ 3) University of Tokyo • Department of Precision Machinery Engineering • Hongo 7-3-1, Bunkyo, Tokyo 113, Japan \\ Tel : +8133812 2111 ex.6449•Fax : +81358006968•Email: higuchi@intellect.pe.u-tokyo.ac.jp
}

Small scaled devices for microparts handling employing an ac electric field boundary wave were proposed in an earlier paper by the authors [1]. These devices, with various novel manipulation features, that instantly generate contactless microparts driving forces through electric field creation, have been designed and produced. In a further attempt, the mechanisms behind microparts conveyance are here subsequently validated in experiments.

Particles as a special group of microparts have been taken as a substitute. On a thin protecting and insulating film interface above a series of encased and insulated parallel field electrodes, particles become either tribo-electrically or induction charged through the application of balanced three- or six-phase voltages. The created non-uniform traveling field-wave conveys the charged particles perpendicular to the electrodes by repulsive forces in a hopping mode from electrode to electrode.

Various particle materials with diameters up to $400 \mu \mathrm{m}$ have been examined; metal, glass, and plastic spheres showed the best performances. The transient charge distribution on particles and on the activated electric device, which is responsible for the particle conveyance, has been statically and dynamically inspected by a modified scanning electron microscope (SEM). Theoretical considerations and simulations underline the experimental findings

In the conclusion of the presentation, a video will be shown.

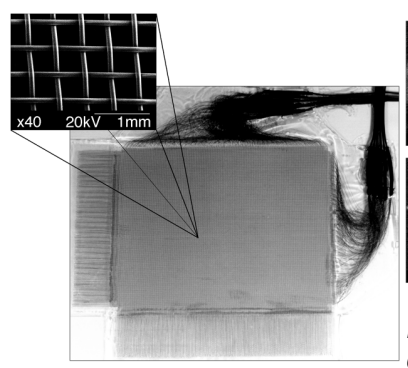
electrodes. Sequence of SEM imticle from right (a) to left (f).

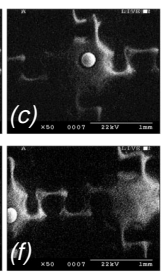

Electric panel with magnified mesh

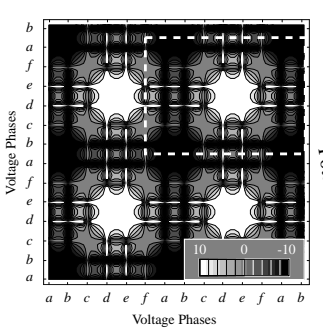

Potential field calculation show ing potential wells on electric panel

device with six-phase voltage application. Particles are confined in the wells and conveyed stepwise over surface.
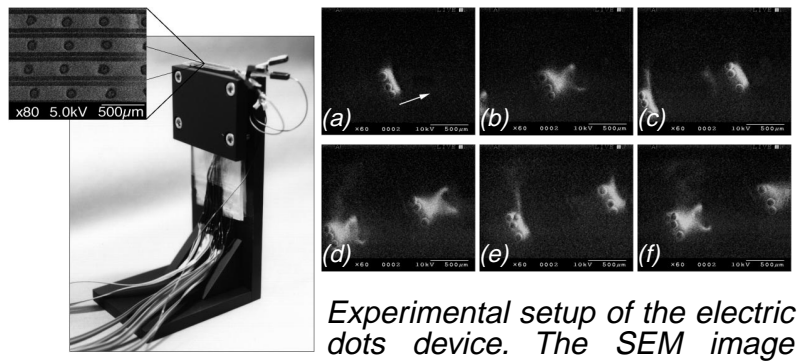

Experimental setup of the electric dots device. The SEM image shows the handling surface with its electrode matrix. Sequence of SEM images visualizes actuation phase-lag.

[1] Moesner, F.M., and Higuchi, T., "Electrostatic Devices for Particle Micro-Handling," IEEE Industry Applications Society, 30th Annual Meeting, Orlando, vol. 2, 10 October, 1995, pp. 1302-1309. 


\title{
Contactless Manipulation of Microparts by ac Electric Fields
}

\author{
Felix M. Moesner ${ }^{1,2)}$ and Toshiro Higuchi ${ }^{2,3)}$ \\ 1) Swiss Federal Institute of Technology Zurich (ETHZ) • Institute of Robotics \\ 8092 Zurich, Switzerland•Email: moesner@ifr.mavt.ethz.ch \\ 2) Kanagawa Academy of Science and Technology $\bullet$ Higuchi Ultimate Mechatronics Project \\ KSP Bldg., East 405, 3-2-1 Sakado, Takatsu-ku, Kawasaki 213, Japan• Fax: +81 448192095 \\ 3) University of Tokyo $\bullet$ Department of Precision Machinery Engineering \\ Hongo 7-3-1, Bunkyo, Tokyo 113, Japan•Email: higuchi@intellect.pe.u-tokyo.ac.jp
}

\begin{abstract}
Small scaled devices for microparts handling employing an ac electric field boundary wave were proposed in an earlier paper by the authors [1]. These devices, with various novel manipulation features, that instantly generate contactless microparts driving forces through electric field creation, have been designed and produced. In a further endeavor, the mechanisms behind microparts conveyance are here subsequently validated in experiments.

Particles as a special group of microparts have been taken as a substitute. On a thin protecting and insulating film interface above a series of encased and insulated parallel field electrodes, particles become either tribo-electrically or induction charged through the application of balanced threeor six-phase voltages. The created non-uniform traveling field-wave conveys the charged particles perpendicular to the electrodes by repulsive forces in a hopping mode from electrode to electrode.

Various particle materials with diameters up to $400 \mu \mathrm{m}$ have been examined; metal, glass, and plastic spheres showed the best performances. The transient charge distribution on particles and on the activated electric device, which is responsible for the particle conveyance, has been statically and dynamically inspected by a modified scanning electron microscope (SEM). Theoretical considerations and simulations underline the experimental findings.
\end{abstract}

\section{Introduction}

In modern industry, there are several implemented techniques employed for handling of particles as microparts. Each technique is suited to a particular goal in the process and also may take advantage of a specific particle property. These handling methods include classical air- and fluidborne transportation by regulated carrier gas or liquid flow. Particles are conveyed in bulk in long flexible tubes and pipes [2]. This purpose-oriented guidance of a mass of particles to a fixed target container, render micro-manipulation of single objects nearly impossible. Apparatuses incorporating this technique form the group of parts feeder which are conceived purely for mass transportation and not for the controlled micro-manipulation of objects.
In contrary to above methods, it is conceivable to use tiny grippers or shovels for single particle manipulation. The goal of these systems is to handle and assemble micro structures. A solution is the grasping and holding of small particles or cells by using microgrippers [3]. Further, a nanorobotic system which was assigned the task of automated handling of micro-parts with nanometer resolution has been developed [4]. This goal-oriented method allows manipulation of single particles despite their different properties.

Listed methods are related by the common application of particle handling. However, they are all together restricted to a particular range of handling abilities, such as bulk transportation, single handling or property-oriented manipulation. With the appropriate use of electrostatic forces however, it is possible to create very compact devices for particle handling, possessing advantages over the above mentioned methods and therefore providing an interesting alternative.

The method of using electrostatic forces for particle handling has already been outlined in various papers and proven in applications. dc electrostatic forces were engaged in surface cleaning and particle dispenser processes [5]. Transportation of fine particles by a three-phase traveling electric field was originally proposed by Masuda in the early seventies [6]. The discovery has been reported as an electric curtain, where aerosol particles are continuously levitated against gravity. Frequency and phase-lag effects on fine particle transportation have been theoretically studied for the electric curtain mode [7], where systems with wide electrode gaps have been proposed. Linear motion of microscopic objects and manipulation of cells are presented in other papers [8,9].

This paper focuses on devices for particle manipulation in the interface between the micro- and macro-world by making use of ac electric fields as the contactless particle conveyor. The electric fields are perpendicularly applied in order to produce confinement of the particles. Two different device designs are employed. 


\section{Principle of Conveyance}

To explain the traveling wave principle, only slim and long parallel electrodes with a constant gap, which form the skeleton of the electric panel, shall be considered in the following interpretation.

Balanced, multi-phase high voltages from a programmable source are supplied to a series of encased electrodes which are covered and protected against electrical breakdown by a thin insulating film, as shown in the cross-sectional view of Fig. 1.

Upon the activation of the electrodes, a traveling electric field is created around the electrodes which is transiently changing in sync with the applied voltage phase. Particles on the insulating film become, depending upon the material, either tribo-electrically or induction charged through the voltage application. Dynamic forces from the created non-uniform traveling field-wave overcome the adhesion and gravitational forces of the charged particles and convey them in a direction in the plane perpendicular to the electrodes in a hopping mode from electrode to electrode.

The supply sequence from the voltage source to the electrodes is given in Table I. Considering balanced rectangular profile voltages, the sequence is written with $[+],[0]$, and [-] for a three- and a six-phase supply. The six-phase case requires all of the 6 attachments, whereas in the threephase case, $a$ and $d, b$ and $e, c$ and $f$ are connected to the same phase. Particle transportation occurs with the variation of the sequence, i.e. the particle synchronously moves with one phase.

Particles involved in the experiments were in the $5 \mu \mathrm{m}$ to $400 \mu \mathrm{m}$ diameter range. Various metals, glass, and plastic spherical particles revealed the best results in a series of conveyance tests. A selection of 70 different types of substances have been examined, and about half of the tested particle materials showed an acceptable frequency-dependent transportation behavior. Representative samples are shown in Fig. 2. It has been observed that spherical particles smaller in diameter than the pitch-length were suitable for conveyance in most of the experiments. For the following experiments, we focused on the usage of spherical, metal and glass particles.

\section{Electric Panel Devices}

The electric panel device is composed of parallel electrodes encased in epoxy resin, a covering thin PET-film, a supporting panel, and electrode-attachments to the phases of the power supply.

Fig. 3 shows the perspective view of an electric panel for bi-directional particle transportation. The particles are

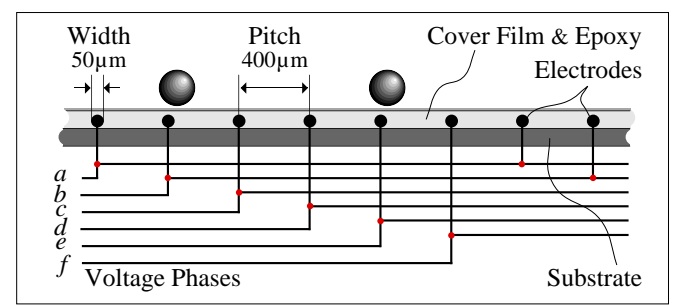

Fig. 1: Cross-section of the electric panel introducing its structural composition. Every sixth electrode is attached to the same phase.

Table I:

6 electrode attachments $a, b, c, d, e, f$ and their supply sequence from a multi-phase voltage source.
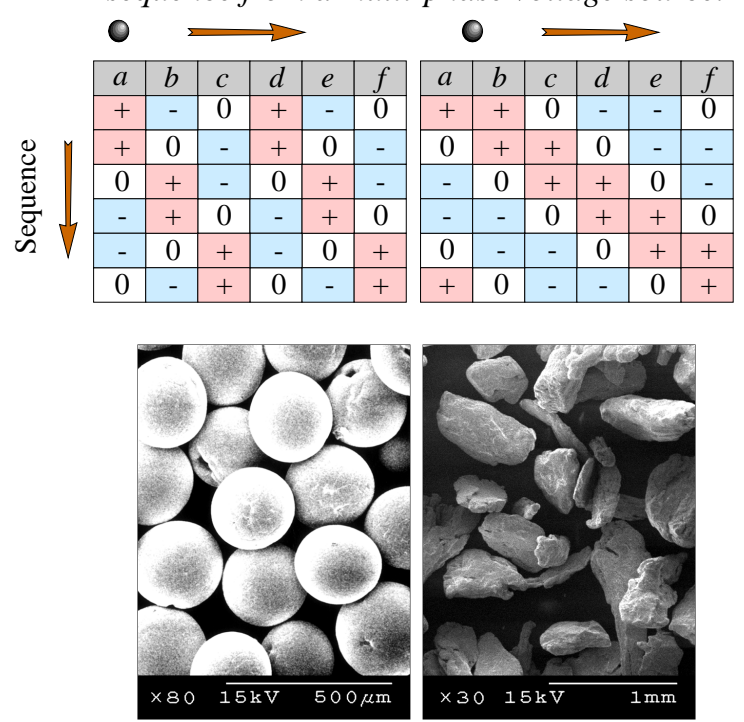

Fig. 2: A SEM micrograph of representative spherical and rugged iron particles.

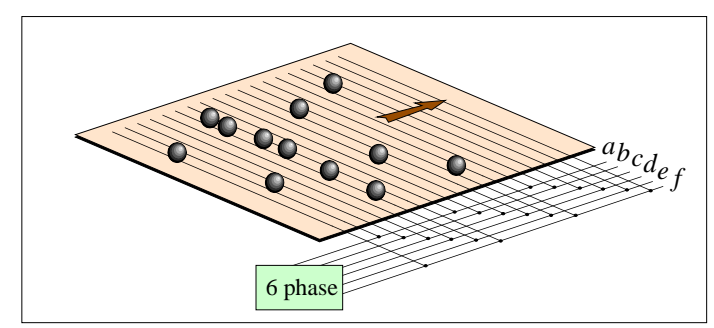

Fig. 3: Electric panel for bi-directional particle transportation perpendicular to the electrodes.

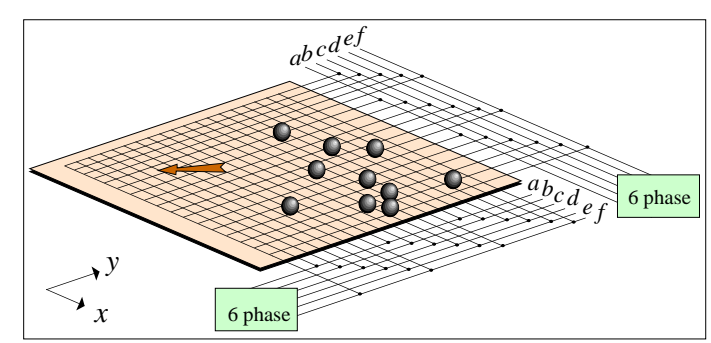

Fig. 4: Electric panel for tetra- and octal-directional particle handling. Highly insulated electrodes are woven to a mesh. 
conveyed perpendicular to the electrodes in one direction across the entire surface and by reversing the voltage supply sequence, the particles are transported in the opposite direction.

An approach to achieving multi-directional particle handling is shown in Fig. 4. Particles can be conveyed twodimensionally over the entire surface; this includes tetradirectional motion when the two electrode sets are activated separately. Octal-directional manipulation is reached by the combined energizing of the two sets.

Because of high voltage differences at the nodal points which can reach $666 \mathrm{kV} / \mathrm{cm}$, individual mesh-electrodes must be covered with a sufficient insulation such as polyamide-imide. This insulator assures a dielectric strength of up to $2756 \mathrm{kV} / \mathrm{cm}$. A picture of the real electric panel is shown in Fig. 5. The field electrodes possess a voltage supply at one end and remain insulated at the other. By this structure, the electrodes function as capacitors with a capacitance of approx. $260 \mathrm{nF}$ in the employed frequency range. The total particle manipulation surface has a dimension of $100 \times 75 \mathrm{~mm}^{2}$. A section of the surface is magnified and represented as a SEM image. It illustrates the mesh structure of the field electrodes. The entire panel is covered by a thin film and hermetically sealed by epoxy resin in order to increase safety during operation.

\section{Potential Distribution of Electric Fields}

In experiments with particles, panels are employed in various actuation modes such as three- and six-phase. It is now of interest to obtain the potential distribution of the electric fields on an electric panel for the six-phase case. The focus of the following calculation is to develop a good agreement between simulation and experimental results.

\subsection{Calculation}

Finding the potential distribution in the region between two conductors, given the charge distribution on the surfaces of the conductors or the potentials of the conductors or a combination of the two, is a problem for which Laplace's equation (1) can be used. In our case, we consider the solution of the two-dimensional Laplace's equation (2).

$$
\begin{gathered}
\nabla^{2} V=0 \\
\frac{\partial^{2} V}{\partial x^{2}}+\frac{\partial^{2} V}{\partial y^{2}}=0
\end{gathered}
$$

Generally, an equation of this type may be solved by the analytical technique, the separation of variables. However, it has been considered to employ a numerical solution in

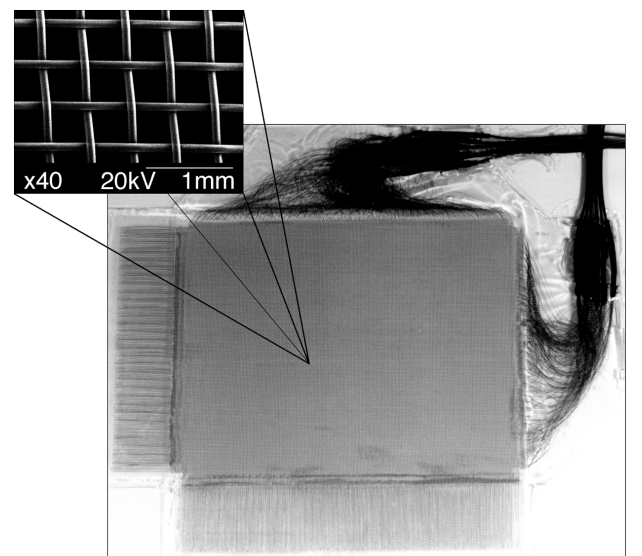

Fig. 5: Electric panel with magnified mesh electrodes.

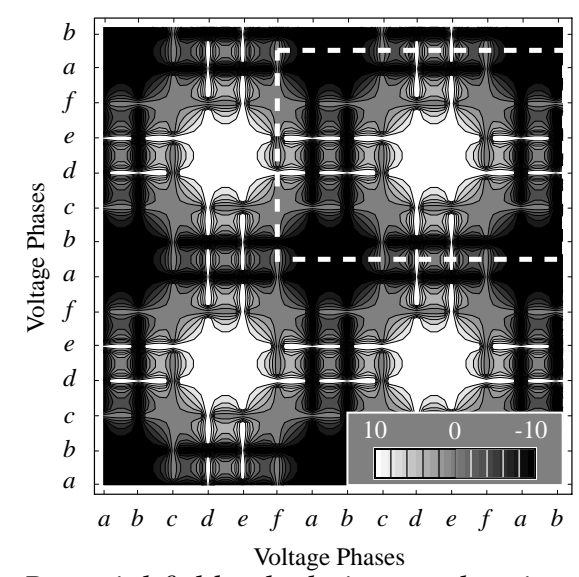

Fig. 6: Potential field calculation on electric panel with six-phase voltage supply.

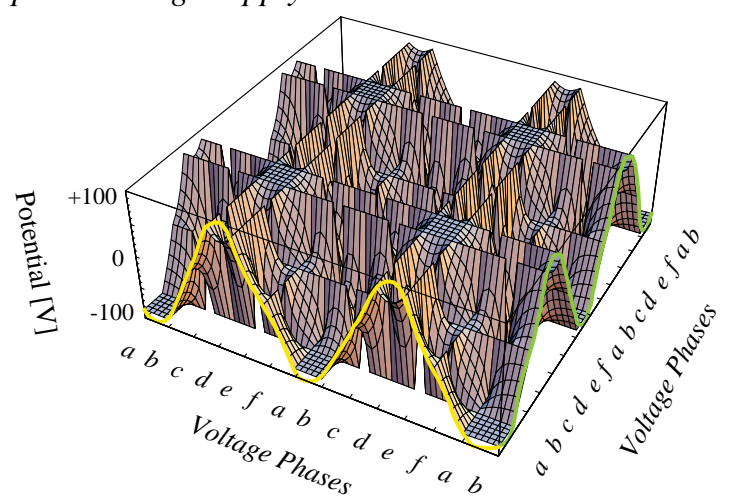

Fig. 7: Six-phase potential wells on electric panel.
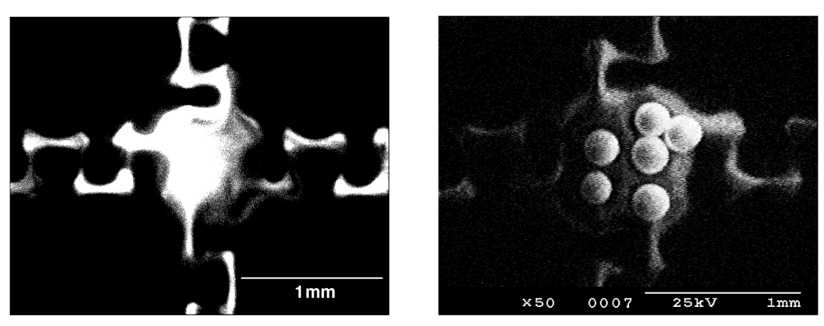

Fig. 8: SEM photos of an activated electric panel. The negative potential well is indicated by white color wherein particles are confined (right photo). 
order to take advantage of fast computers (Sun Ultra 1). The method employed is derived from the finite-difference approximation [10].

14 mesh-electrodes of the electric panel have been defined in a table and virtually connected to their appropriate phase $a, b, c, d, e$, and $f$. The table is traversed iteratively by means of the finite-difference approximation until the computed error is smaller than a defined constant.

\subsection{Results}

The computational calculations of the potential distribution is shown in Fig. 6. The result is obtained by a potential distribution calculation of a six-phase voltage supply to the electrodes. As a result, a checker board pattern is obtained. The white and black areas represent the $10 \mathrm{~V}$ and $-10 \mathrm{~V}$ potential level, respectively. Another view is shown in Fig. 7, where potential wells are clearly visible.

The two SEM micrographs of Fig. 8 depict the charge distribution on an activated electric panel. The SEM works as a charge distribution sensor. The white pattern represents negative surface charge; positive charge cannot be detected by the SEM. Since charge and potential is considered to be analogous, the SEM images can be compared directly with the potential distribution calculation. A white frame has been inserted in Fig. 6 to visualize the area where the SEM image fits into. It is not difficult to apprehend a good agreement with the overall pattern-structure.

The potential calculation in Fig. 6 corresponds very well with the checker board pattern of conveyed particles in Fig. 9. The white frame visualizes the location which the computed graphic of Fig. 6 matches. The photograph does not disclose particles which are positively or negatively charged. They are confined in the potential wells visualized in Fig. 7.

\section{Observation of Particle Conveyance}

The usage of a SEM also allows the dynamic observation of traveling charge distribution and particle conveyance. The motion of a single particle is captured in the TV mode. As a result, the sequence in Fig. 10 shows the particle following the traveling field wave of a six-phase supply. It should be noted, that the tracked conveyance technique is employed [1]. Hereby, the track confines the particle on a line, which is visible as a static charge distribution. The perpendicular electrodes actuate the particle from right (a) to left (f). The sequence reveals how the particle moves step by step over 6 electrode-pitches. This sequence illustrates two facts: first, the particle is conveyed by the electric field of the ac activated electrodes and second, the particle is confined on a line by the dc energized electrodes.

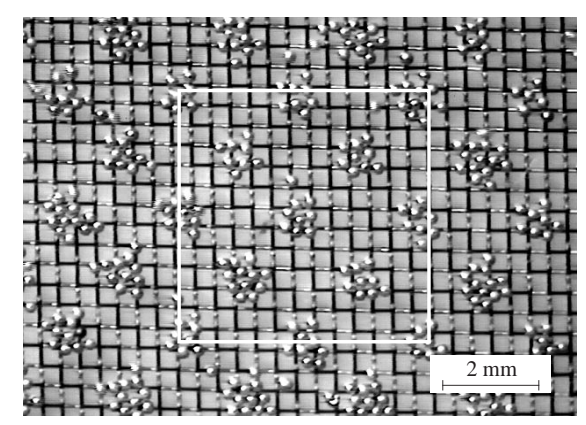

Fig. 9: Checker board pattern: Groups of particles are confined in potential wells.

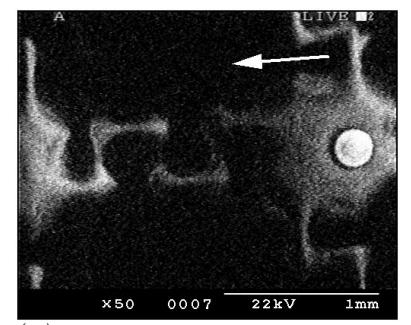

(a)
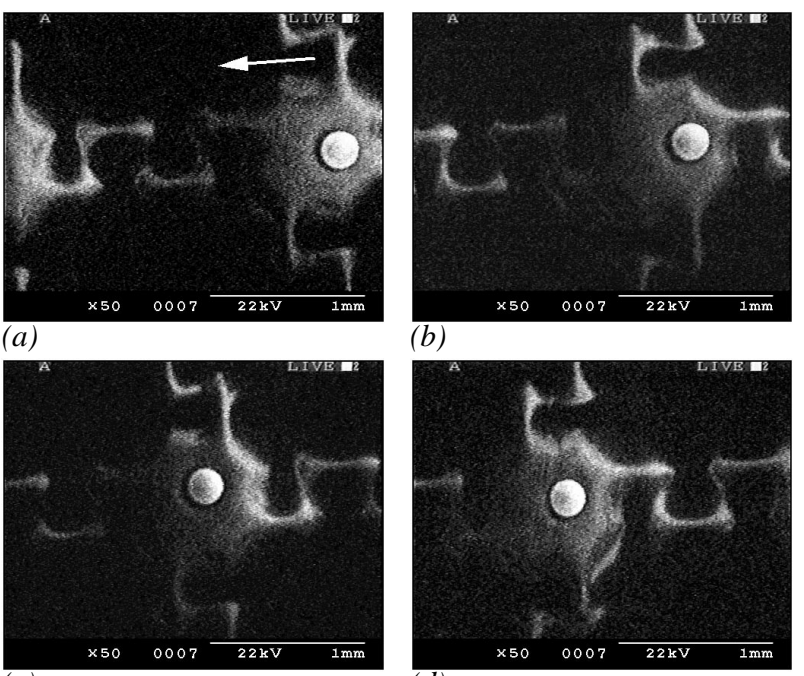

(b)

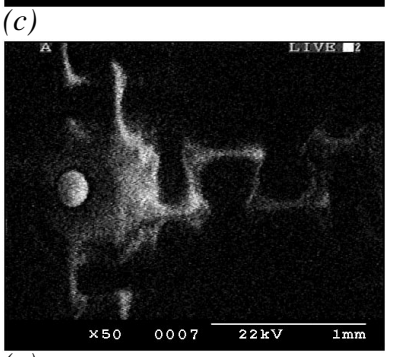

(e)

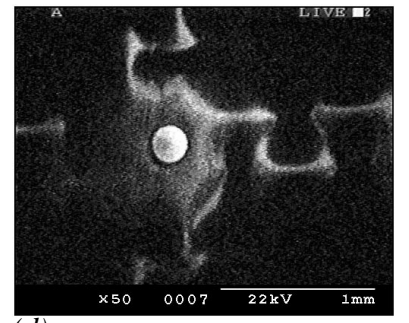

(d)

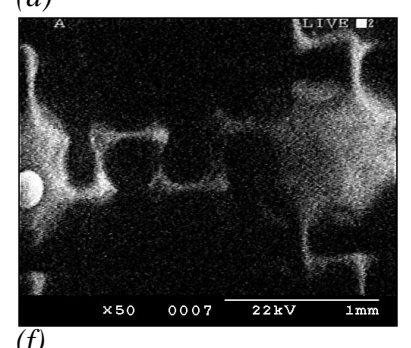

(f)

Fig. 10: Sequence of SEM images revealing tracked conveyance of a single particle from right (a) to left $(f)$.

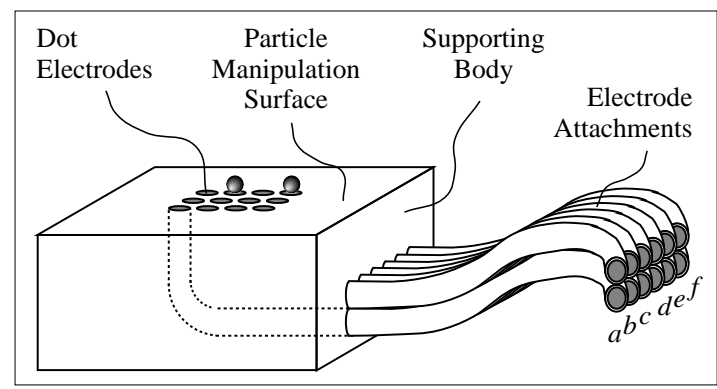

Fig. 11: Perspective of the electric dots device. The field electrodes are embedded in a epoxy resin body and connected to their phases. 


\section{Electric Dots Devices}

\subsection{Fabrication}

The electric dots and panel device consist of a very similar structure and are functionally closely related. The main difference is the shape of the field electrodes. They are designed as long parallel electrodes in the panel, but as pinpoint electrodes in the other device. The effect of the traveling electric field is the same, but the positioning of the particles is more individual and precise with the dot matrix. Fig. 11 illustrates the structure of the dot matrix device. All electrodes are embedded in epoxy resin. Their terminal ends are cut perpendicularly and form, together with the body, the manipulation surface. The individual field electrodes are connected to their corresponding voltage phase. In practice, a sandwich structure of neatly cut electric panel layers delivers the desired dot electrodes in a matrix configuration. The SEM photo in Fig. 12 depicts the dot electrodes in the manipulation surface, which are visibly composed like a sandwich. Our realized matrix has a $(6 \times \mathrm{n})$-dimension, i.e. it consists of 6 rows as shown in Fig. 13. The two central rows are responsible for particle actuation and the remaining four peripheral rows provide a potential track for the particles through their dc activation. The dots in Fig. 13 are horizontally activated allowing the wave to travel either from left to right or vise versa.

\subsection{Surface Charge \& Potential Distribution}

The SEM delivers an image of the negatively charged areas on the manipulation surface, as shown in Fig. 14a for the six-phase application case. Compared with the corresponding picture of previous Fig. 13, the particles are confined in the activated locations. Again, the simulation of the potential distribution in Fig. 14b provides a good agreement with the SEM image. The potential wells portrayed in Fig. 14c produce a good understanding of the particle confining mechanism.

\subsection{Phase Lag}

One effect which has been observed in experiments and theoretically described in [7] is the phase-lag between the advancing traveling electric field and the conveyed particle. It has been noted that the particle still remains at a location while the traveling field wave is proceeding. A dynamic sequence of SEM images is shown in Fig. 15. It exposes the close-up actuation of a group of $100 \mu \mathrm{m}$ glass particles in the direction of the inserted arrow on the manipulation surface of an electric dots device. A threephase electrode activation has been chosen to pay attention to this effect in more detail.

In (a) and (b), the particles remain on a particular spot while the field travels to the next location; then the parti-

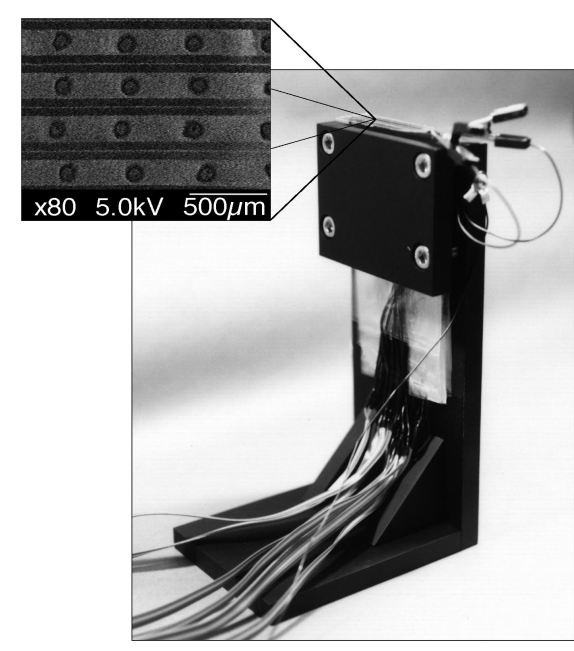

Fig. 12: Experimental setup of the electric dots device. The handling surface is at the top and magnified by a SEM image revealing its matrix dot electrodes.

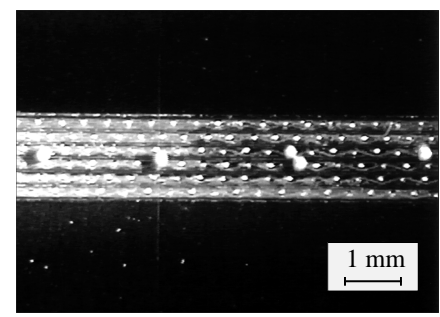

Fig. 13: Confined particle conveyance. Six-phase voltage wave transports particles along an array of electrodes.

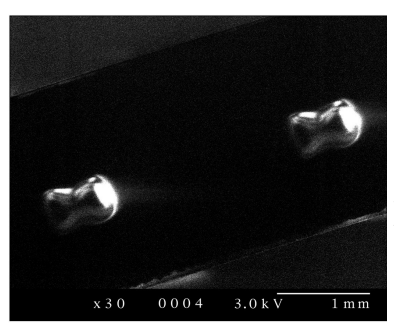

(a)

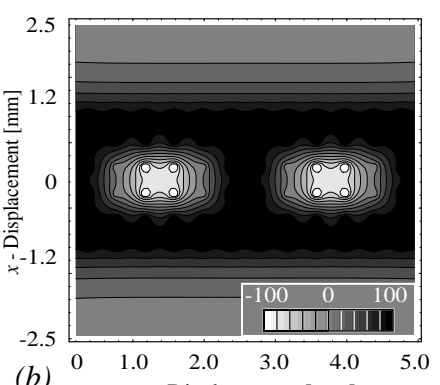

(b) $y$ - Displacement $[\mathrm{mm}]$

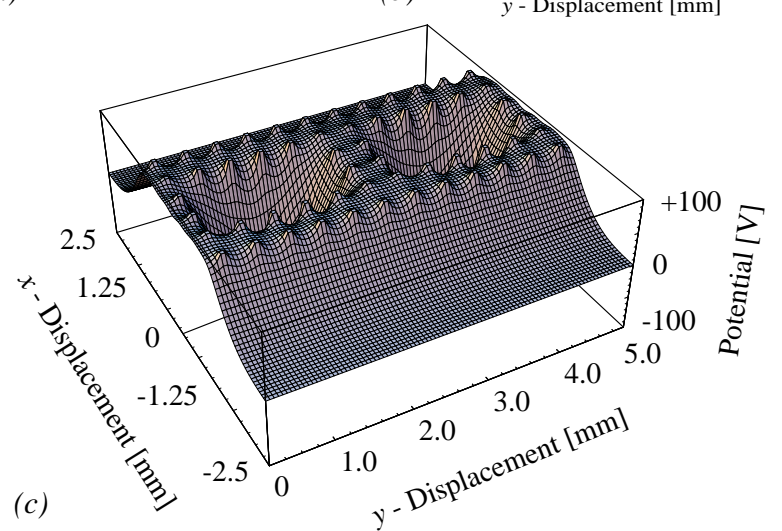

Fig. 14: SEM photo $(a)$ and simulation $(b, c)$ with analogous structural dimensions and six-phase voltage supply. The potential wells clearly appear in (c) as cavities. 
cles follow (c) and stay again (d) while the field is proceeding. Finally, they follow on as shown in (e). This effect appears as particles try to find an equilibrium between the transiently changing repulsive and attractive forces of the traveling field-wave.

\section{Conclusion}

For the electric panel and dots device, the potential distributions of electric fields in a six-phase mode have been calculated and validated with charge distribution SEM images and conventional microscope photos. The results show very good agreement. The useful validation offers an insight behind the confined particle manipulation.

The dynamic particle conveyance mechanism has been further explained by two SEM image sequences where the phase-lag between traveling wave and particle is additionally commented.

\section{Acknowledgment}

We wish to acknowledge the appreciated and valuable collaboration of our colleagues S. Yashiro for his engagement in the electric panel devices, S. Egawa and T. Niino for their expertise on voltage supply systems, J. Jin and S.J. Woo for prolific discussions, and Y. Tanii and W. Yang for their contributory involvement in this project.

\section{References}

[1] Moesner, F.M. et al., "Electrostatic Devices for Particle Micro-Handling," IEEE Industry Applications Society, 30th Annual Meeting, Orlando, vol. 2, 10 October, 1995, pp. 1302-1309.

[2] Masuda, H. et al., "The Static Electrification of Particles in Gas-Solids Pipe Flow," AlChE Journal, vol. 22, no. 3, May 1976, pp. 558-564.

[3] Greitmann, G. et al., "Tactile Microgripper for Automated Handling of Microparts," Sensors \& Actuators: A. Physical, vol. SNA 053 / 1-4, 1996, pp. 409-414.

[4] Codourey, A. et al., "A Robot for Automated Handling in Micro-World," IROS'95 IEEE/RSJ, Pittsburgh, Pennsylvania, August 1995, pp. 185-190.

[5] Cooper, D.W., et al., "Surface Cleaning by Electrostatic Removal of Particles," Aerosol Science and Technology, vol. 13, 1990, pp. 116-123.

[6] Masuda, S., et al., "Confinement and Transportation of Charged Aerosol Clouds via Electric Curtain," Electrical Engineering in Japan, vol. 92, no. 1, 1972, pp. 43-52.
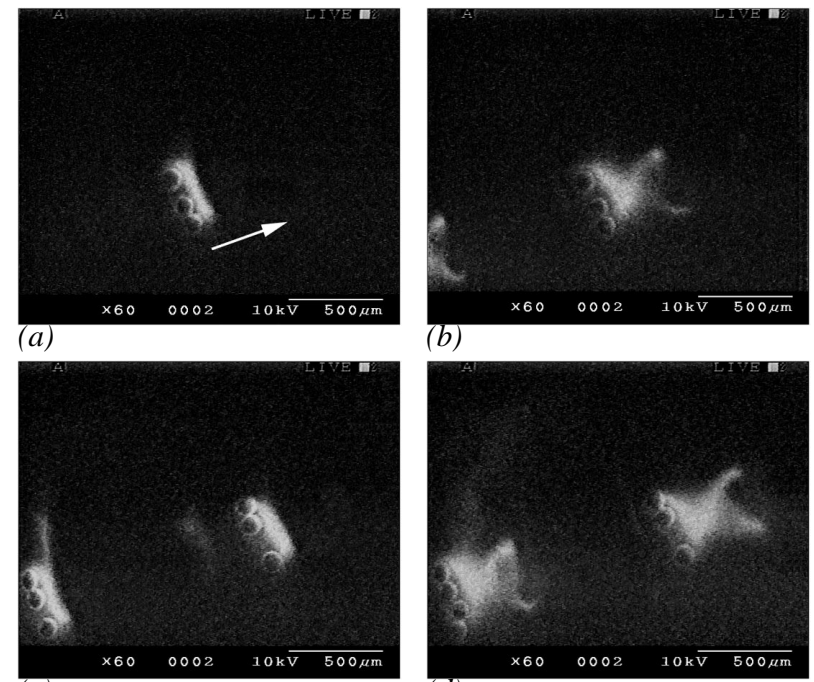

(c)
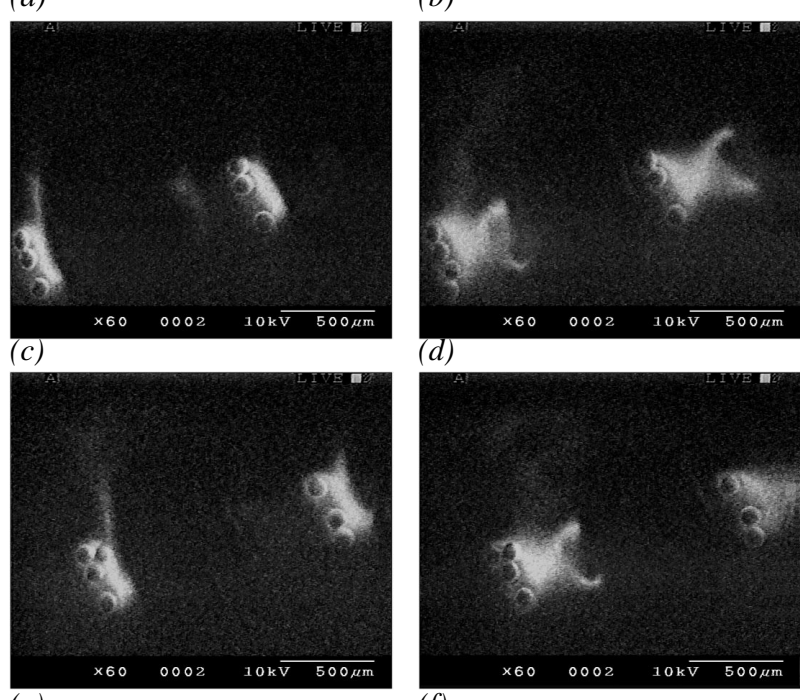

(d)

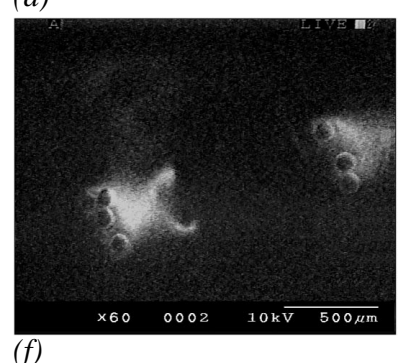

(f)

Fig. 15: Dynamic sequence of SEM images revealing the actual phase-lag between traveling field and conveyed particle. The glass particles show an actuation in direction of the arrow.

[7] Gan-Mor, S. et al., "Frequency and Phase-Lag Effects on Transportation of Particulates by an ac Electric Field," IEEE Transactions on Industrial Applications, vol. 28, no. 2, March 1992, pp. 317-323.

[8] Fuhr, G. et al., "Linear Motion of Dielectric Particles and Living Cells in Microfabricated Structures induced by Traveling Electric Fields," Proc. IEEE Micro Electro Mechanical Systems Workshop, Nara, Japan, January / February 1991, pp. 259-264.

[9] Pethig, R., "Applications of ac Electrical Fields to the Manipulation and Characterisation of Cells," Conference Record 4th Toyota Conference, October 1990, pp. 159-185.

[10] Rao, N.N., "Elements of Engineering Electromagnetics," Prentice-Hall Inc. Fourth Edition, 1994. 